\title{
Performance Evaluation of Linear Beamforming Receiver for Large CoMP Sparse Massive MIMO Channel Matrices
}

\author{
Amir M. Ahmadian*†, Rakash Sivasiva Ganesan*, Wolfgang Zirwas* \\ *Nokia Bell Labs, Radio Systems Research, Munich, Germany \\ ${ }^{\dagger}$ Institute for Communications Engineering, Technische Universität München, Munich, Germany \\ E-mail: \{amir.ahmadian_tehrani.ext, rakash.sivasivaganesan, wolfgang.zirwas\}@nokia-bell-labs.com
}

\begin{abstract}
A massive multiple-input, multiple-output (mMIMO) downlink is considered with fixed wideband beams known as Grid of Beams (GoBs). The radio channel of a typical urban macro scenario will be shown to be sparse, and in combination with a linear maximum ratio combining beamforming method is used at the user equipment. Simulation results for urban macro cells show that reasonable spectral efficiencies are achieved with a moderate number of relevant channel components (RCCs) and accordingly limited feedback overhead for reporting of channel state information (CSI).
\end{abstract}

Index Terms-Maximum Ratio Combining, Receiver Beamforming, massive MIMO

\section{INTRODUCTION}

Massive multiple-input, multiple-output (mMIMO) multiuser MIMO is a key candidate for $5 \mathrm{G}$ systems to handle orders of magnitude more data traffic and to improve power efficiency as well as to reduce inter cell interference by means of directed transmission of energy [1], [2], [3]. However, the large number of antennas results in a high overhead for channel state information estimation as well reporting in case of frequency division duplex systems (FDD). FDD massive MIMO literatures concentrates on compressing the CSI feedback load by exploiting the spatial correlations [4]. Further, exploiting the joint sparsity structure among the coscheduled UEs reducing the feedback overhead has been taken into attention [5].Grid of beams (GoBs) has been proposed to reduce feedback by limiting the transmit signals to a set of narrow fixed beams [6]. Still, the high level of sensitivity to channel estimation errors for accurate multi user MIMO precoding as well as limited CSI reporting overhead continues to provide considerable challenges. Thus, a flexible and adaptive CSI reporting scheme is needed. Previously proposed techniques like the GoB has reduced the number of reporting coefficients and the precoder complexity [7]. In this paper we extend the single antenna UE CSI feedback to multiple UE antenna reporting for the "relevant" channel components, which we define as the effective channel matrix coefficients that are above a certain power threshold compared to the strongest channel component per UE.

This paper is organized as follows. Section II provides a brief overview of the scheme. In Section II, we discuss the

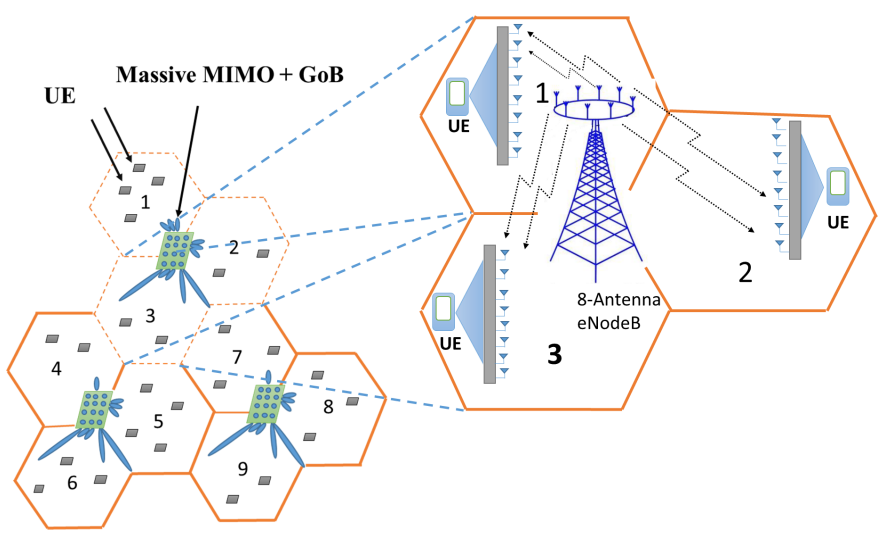

Fig. 1: System model.

system model, and in Section III, we provide simulations to show gains. The paper concludes with Section IV.

\section{SySTEM MOdeL}

Consider the urban macro cooperation area scenario described in [7] that uses the according 3GPP 3D channel model [8]. The Tx-beams are generated through a fixed grid of beam $(\mathrm{GoB})$ and interference can be overcome in combination with joint transmission coordinated multipoint (CoMP) featuring adjust sites synchronization, synchronous exchange of user data, and channel state information (CSI) reporting. Suppose each eNodeB generates eight beams in the azimuth direction and two in the elevation direction. With two polarizations per beam, we have 32 beams per antenna element or $32 \times 8$ beams per cell (See Fig. 1).

We define a relevant channel component (RCC) to be an effective radio channel of the link between eNodeB beam and UE beam used for CSI feedback reporting. GoB can provide efficient feedback by reporting only the relevant channel components within a threshold value with respect to the strongest channel matrix component seen by the UE. We calculate the channel precoding matrix $\mathbf{W}$ by using the pseudo inverse of the sparse effective channel matrix $\mathbf{H}$ based on reporting of the relevant RCCs above the power threshold relative to the strongest beam. 


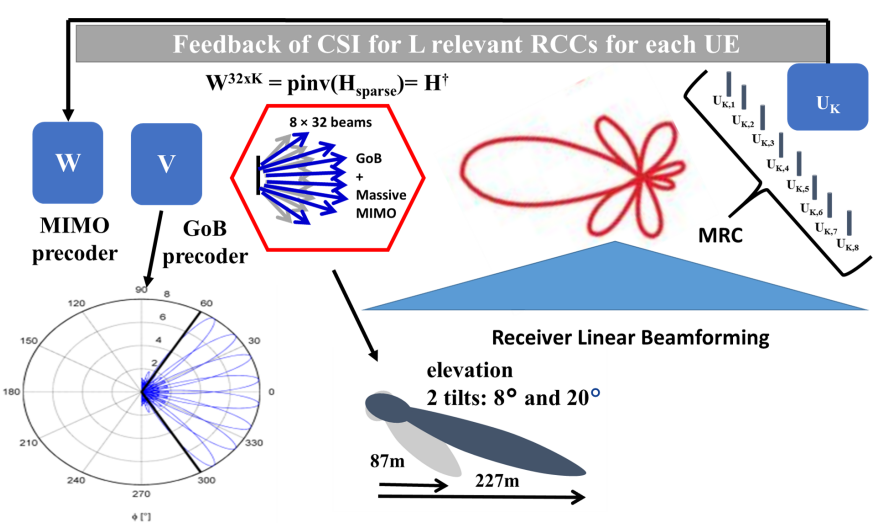

Fig. 2: System model beamforming per user per cell.

For 5G we assume that UEs might be equipped with up to eight antenna elements and in the simplest case a maximum ratio combining (MRC) Rx-beamformer can be applied with respect to the strongest Tx-beam. The MRC weight to combine the strongest Tx-beam $b_{n}(i)$ of $i_{t h} \mathrm{UE}$ at $n_{t h}$ antenna to the channel component $h_{n}(i)$ is given by a complex correlation between the two signals:

$$
\omega_{n}(i)=b_{n}(i)^{*} h_{n}(i),
$$

where $\omega_{n}(i)$ is the MRC weight for Tx-beam $b_{n}(i)$ and (.)* denotes a complex conjugate transpose. The MRC output for the $n_{t h}$ antenna at $i_{t h} \mathrm{UE}$ is then given by summation of all the weighted signals as:

$$
h_{M R C}(i)=\sum_{n=1}^{8} \omega_{n}(i) b_{n}(i) .
$$

Obviously, the same beamforming weights will affect the reception of all other beams from the same UE. Generally the Rx-beamformer for the strongest Tx-beam will have a mismatch to the other $288 \mathrm{Tx}$-beams of the cooperation area so that the relative Rx-power of these beams will be reduced. Assuming a fixed threshold for the definition of relevant beams this means that MRC beamformers at the UEs can reduce the number of RCCs. Thus, adding a UE MRC beamformer limits CSI reporting overhead due to a lower average number of channel components compared to a single omni-dimensional Rx antenna. This statement will be exemplified in Section III.

\section{System Level Simulation Model}

We consider a JT CoMP inter-site cooperation area of size $1320 m \times 1160 m$ consisting of three sites with three sectors, i.e., of nine cells. The $3 \mathrm{D}$ channel model is according to the 3 GPP urban macro scenario forming a 32 beam GoB [8]. UEs are randomly dropped into the cooperation area and served from the cell with the strongest among all the received beams.

Fig. 3 illustrates the UE and eNodeB positions, the three selected sites as well as the cell locations. The inter-site distances are 500m. Fig. 4 shows the 90 UE (10 UEs per cell) positions that were randomly selected with a uniform

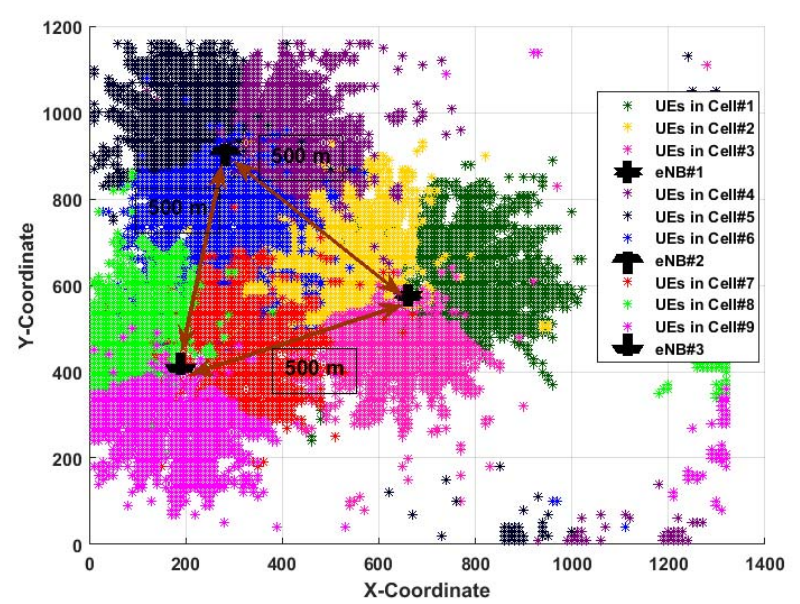

Fig. 3: UEs distribution in three inter-site urban macro cells scenario .

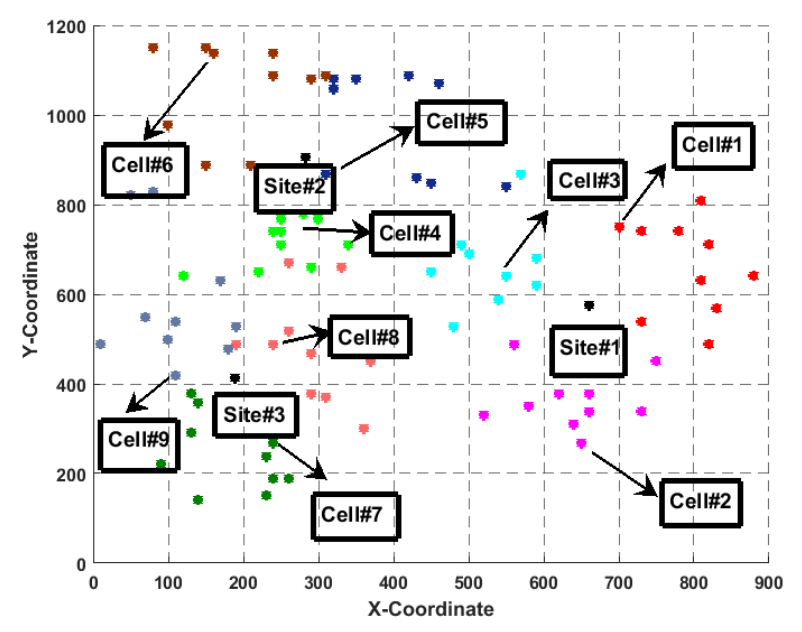

Fig. 4: Random selected UEs in three inter-site urban macro cells scenario.

distribution over the cooperation area. Using the geometrybased 3D channel model the GoB is obtained by a first fixed precoder applying two different antenna tilt values (7 and 18 degree) to 8 azimuth beams with two polarizations each. (See Fig. 5):

1) UEs are allocated to cells based on the strongest received beam. In other words, UEs located in the first cell have the beam with the highest received power in the first cell.

2) next randomly 10 UEs per cell are selected so that for a three site cooperation area there will be 90 UEs distributed in the 9 cells.

3) Path loss - like e.g. RSRP - measurements for all Txbeams are collected per UE. With 32 beams per cell and nine cells per cooperation area there are $32 \times 9=288$ channel components in total per UE. 
4) Once the channel components are collected at the UE, a Fast Fourier Transform (FFT) of size 2048 is operated for each Rx-signal for each of the eight UE antenna elements.

5) The frequency domain signal is created after the FFTs is limited to to a $18 \mathrm{MHz}$ bandwidth a $1200 \mathrm{sub}$ carrier or 100 physical resource blocks (PRB) of size $12 \mathrm{sub}$ carriers times 14 OFDM symbols.

6) Applying the proposed maximum ratio combining method at the UE (see Section III) over 8 receiver antenna elements, the maximum ratio combined effected channel matrix is obtained.

\section{A. Performance metrics and analysis}

To exemplify the impact of using UE MRC beamformer and effected channel matrix components reduction by reporting RCCs with respect to the strongest beam on the system level performance, spectral efficiency as well as power normalization loss (PNL) of single UE antenna channel matrix $\mathbf{H}$ in contrast with eight UE antenna channel matrix $\mathbf{H}_{M R C}$ have been evaluated. Using zero forcing beamforming (ZFBF) as an interferece zero forcing objective linear precoder and similarly to single antenna UE precoder $\mathbf{W}$, we have:

$$
\mathbf{W}_{M R C}=\mathbf{H}_{M R C}^{T}\left(\mathbf{H}_{M R C} \mathbf{H}_{M R C}^{T}\right)^{-1},
$$

where $\mathbf{H}_{M R C}$ denotes eight UE antenna channel matrix and $\mathbf{H}_{M R C}^{T}$ represents the transpose of eight UE antenna channel matrix respectively. As such, the MRC beamformed received signal $\mathbf{y}_{M R C}$ can be assumed as follows:

$$
\mathbf{y}_{M R C}=\mathbf{H}_{M R C} \mathbf{W}_{M R C} .
$$

The essential simulation parameters are summarized in Table I. Transmit power per cell is equally distributed over 1200 subcarriers and 32 beams with used bandwidth of $18 \mathrm{MHz}$ around a carrier frequency of $2.1 \mathrm{GHz}$. The derived signal-tointerference-plus-noise ratio (SINR) per UE can be expressed as [7]:

$$
\begin{array}{r}
S I N R[\mathrm{~dB}]=\mathrm{y}_{U E(i)} P_{t x}[\mathrm{dBm}]- \\
\mathrm{P}_{n}[\mathrm{dBm}]-\mathrm{I}_{U E(i)}-\mathrm{PNL}_{U E(i)}[\mathrm{dB}],
\end{array}
$$

where

$$
\begin{gathered}
P_{t x}=\text { Tx } \text { Power }_{d B m} / N_{U E}-10 \log _{10} N_{s u b} \times N_{P R B}, \\
P_{n}=-173.83+10 \log _{10} N_{S u b S p}+\text { Receiver } N F[\mathrm{~dB}] .
\end{gathered}
$$

$P_{t x}$ in (5) specifies the transmit power distributed equally among all the users which is obtained via (6). $\mathrm{I}_{U E(i)}$ denotes the received interference at the UE with the index $\mathrm{i}$ in $\mathrm{dBm}$. $N_{U E}$ is number of UEs in one cell, $N_{s u b}$ is the number of subcarriers and $N_{P R B}$ is the number of PRBs expressed in (6). In order to observe the effect of noise to the channel, the noise formulation referred to as $P_{n}$ has been used. $N_{S u b S p}$ denotes the subcarrier spacing and Receiver $N F$ refers to the receiver noise figure in $\mathrm{dB}$. Spectral efficiency per cell in bit/sec/Hz/cell for both scenario with single UE antenna and eight antennas UE can be obtained respectively [7]:

$$
S E=\frac{N_{U E} \times C_{L T E} \times N_{s u b} \times N_{O F D M}}{T T I \times U B \times N_{\text {Cell }}},
$$

Where $C_{L T E}$ is the SINR to bit rate mapping based on the 3GPP physical layer procedures described in [9], where the measured signal-to-interference-plus-noise ratio (SINR) is mapped to LTE modulation and coding scheme (MCS). Power normalization loss is considered per UE after according per UE power normalization. Power normalization has to ensure that the overall Tx power per cooperation area of $P_{T x}$ times 9 (for nine cells) is not exceeded and UE individual power normalization ensures fairness between all UEs. Characterizing the performance of overall solution, PNL of $i_{t h}$ UE can be obtained as follows:

$$
P N L(i)_{d B}=10 \log _{10} \sum_{n=1}^{288} P_{W_{U E(i, n)}},
$$

Where $P_{W_{U E(i, n)}}$ is the power of normalized precoder $\mathbf{W}$ of $i_{t h}$ UE over the $n_{t h}$ received beam. Obviously, above expression can be used to calculate power normalization loss for both $\mathbf{W}$ and $\mathbf{W}_{M R C}$.

\section{B. Performance examples}

In what follows, we discuss the numerical results obtained based on the system model detailed above. To exemplify the impact of changing RCCs when each UE is equipped with linear beamforming using 8 received antennas in the cooperation area, two power level thresholds $(-20 \mathrm{~dB},-25 \mathrm{~dB})$ with respect to the strongest channel component per user have been employed.

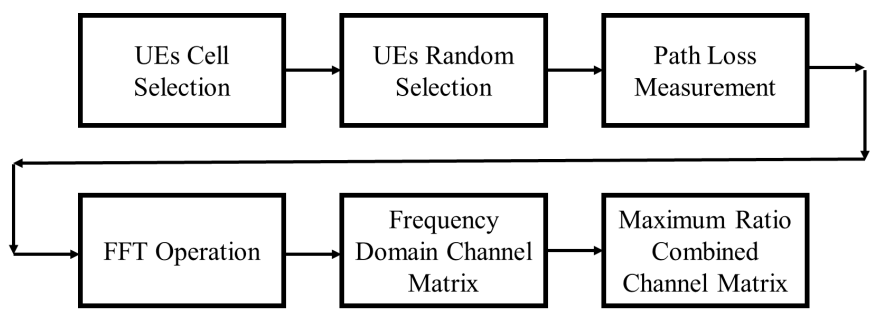

Fig. 5: Maximum ratio combined channel matrix creation.

Fig. 6 contrasts the number of reported relevant channel components to the eNodeB for a single antenna UE against UEs employing MRC beamformers with eight UE antennas. Utilizing UE beamformer leads to maximizing the received power on one hand and reducing the number of reported RCCs down to approximately 50 percent on average on the other hand. Further, this impact more pronounced for the lower threshold value of $-25 \mathrm{~dB}$. Fig. 7 provides the $\mathrm{CDF}$ for the power normalization loss over 100 PRBs.

In order to take scheduling aspects for MU MIMO user grouping into account, UEs are only scheduled in case their PNL is below $5 \mathrm{~dB}$ and otherwise taken out of the MU MIMO group. As it can be seen, the PNL for the case of an ideal 


\begin{tabular}{|c|c|c|}
\hline Symbols & PHY Layer Parameters Value & Value \\
\hline $\mathrm{C}$ & Carrier Frequency $(\mathrm{GHz})$ & 2.1 \\
\hline $\mathrm{TB}$ & Total Bandwidth $(\mathrm{MHz})$ & 20 \\
\hline $\mathrm{UB}$ & Used Bandwidth $(\mathrm{MHz})$ & 18 \\
\hline$N_{\text {SubSp }}$ & Subcarrier Spacing $(\mathrm{KHz})$ & 15 \\
\hline$N_{\text {sub }}$ & Number of Subcarriers & 1200 \\
\hline$N_{P R B}$ & Number of PRBs & 100 \\
\hline Tx Power & Tx Power per per cell $(\mathrm{dBm})$ & 46 \\
\hline Receiver $N F$ & Receiver NF (dB) & 7 \\
\hline$N_{U E}$ & Number of UEs & 90 \\
\hline$N_{\text {Antenna }}$ & Number of Antennas per UE & 8 \\
\hline$N_{\text {cell }}$ & Number of Cells & 9 \\
\hline$N_{\text {Beams }}$ & Number of Tx Beams & 288 \\
\hline$N_{O F D M}$ & Number of OFDM blocks per subframe & 14 \\
\hline TTI & Duration of one subframe (ms) & 1 \\
\hline
\end{tabular}

TABLE I: Simulation Parameters

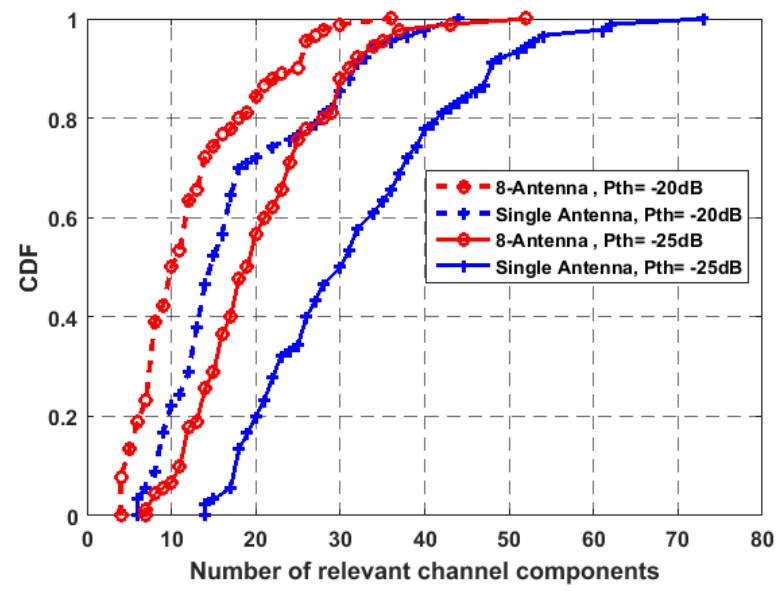

Fig. 6: CDF plot for RCCs per UE.

reporting of all channel components is by roughly one $\mathrm{dB}$ smaller compared to the case of limited reporting of relevant channel components, which is expected due to the lower condition number of the RCC channel matrix.

Fig. 8 compares the spectral efficiency per cell for single antenna UE and 8-antenna UE cases and full CSI for all channel components when different power threshold levels applied to the channel coefficients. As it can be seen, beamforming at the UE using MRC can result in moderate improvement in spectral efficiency going up by roughly 15 percent when $\mathrm{PThr}=-20 \mathrm{~dB}$ and MRC beamformer is used at the UE. Further, the spectral efficiency increases up to 10 percent in both scenarios when full CSI feedback for all channel components is being provided. As such, MRC beamformer leads to maximizing the received power as well as limiting reporting to lower number of relevant channel components. Choosing as appropriate power threshold is important to balance both effects.

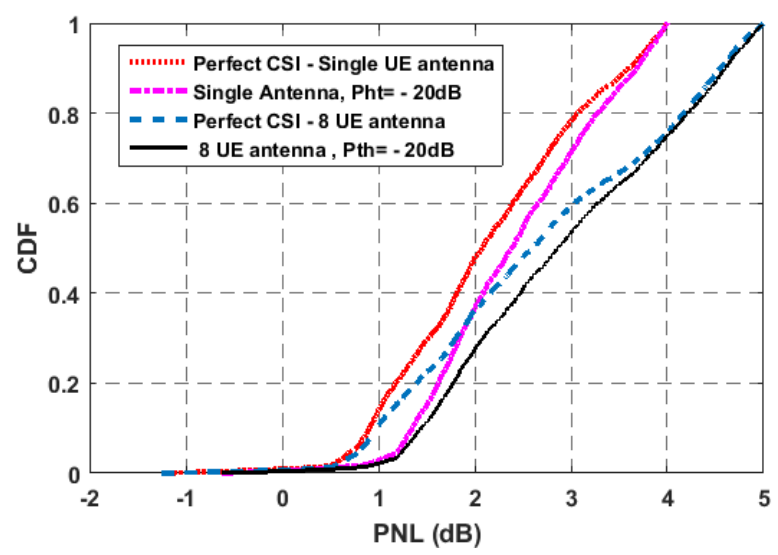

Fig. 7: PNL plot with $7 \mathrm{~dB}$ threshold.

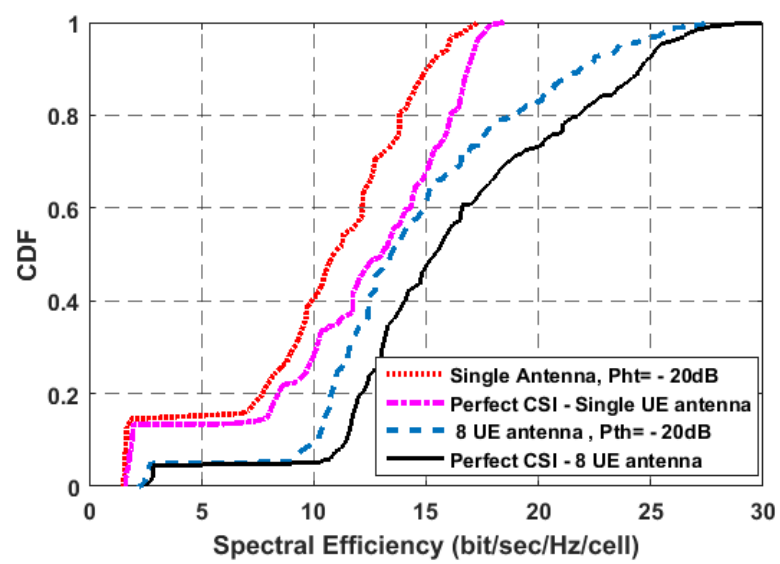

Fig. 8: CDF plot for spectral efficiency per cell .

\section{CONCLUSION}

Flexible and effective CSI reporting is one of the main enablers for future $5 \mathrm{G}$ radio systems featuring concepts like JT CoMP where tight cooperation between network entities requires low effort transmission configurations with minimum reporting overhead. Simulation results for a nine cell cooperation area demonstrates that CSI feedback reporting overhead can be reasonably dropped by using UE beamformer. More specifically, both number of relevant channel components and interference between sites can be moderately decreased when linear beamforming is used at the UE. Moreover, power normalization loss which characterizes the performance based on precoder power imposes much less impact when MRC beamformer at the receiver is used. System level simulation results confirm that we could achieve reasonable spectral efficiency with slight degradation of the precoding performance in combination with moderate CSI feedback overhead by choosing an appropriate power threshold.

\section{ACKNOWLEDGMENT}

Part of this work has been performed in the framework of the Horizon 2020 project FANTASTIC-5G (ICT-671660), 
which is partly funded by the European Union. The authors would like to acknowledge the contributions of their colleagues in FANTASTIC-5G, although the views expressed are those of the authors and do not necessarily represent the project.further, authors of this paper would like to thank Professor Gerhard Kramer, Institute for Communications Engineering, Technische Universität München, Munich, Germany for his support and time invested for this work.

\section{REFERENCES}

[1] E. Bjornson, E. Larsson, and T. L. Marzetta, "Massive mimo: ten myths and one critical question," in IEEE Communications Magazine, February 2016.

[2] 3GPP, “TR 36.897: Technical specification group radio access network: study on elevation beamforming/ full-dimension (FD) multiple input multiple output (MIMO) for LTE (Release 13)." in 3GPP, Thech. Rep. V13.0.0,2015

[3] T. L. Marzetta, "Noncooperative cellular wireless with unlimited numbers of base station antennas," IEEE Transactions on Wireless Communications, vol. 9, no. 11, pp. 3590-3600, November 2010.
[4] J. Nam, J. Y. Ahn, A. Adhikary, and G. Caire, "Joint spatial division and multiplexing: Realizing massive MIMO gains with limited channel state information," in Proc. of the 46th Annual IEEE CISS, September 2012.

[5] X. Rao and V. K. N. Lau, "Distributed compressive csit estimation and feedback protocols for fdd multi-user massive MIMO systems," IEEE Transactions on Signal Processing, vol. 62, no. 12, pp. 3261-3271, 2014.

[6] T. Obara, S. Suyama, J. Shen, and Y. Okumura, "Joint fixed beamforming and eigenmode precoding for super high bit rate massive MIMO sytems using higher frequency bands," in Proc. of the 25th Annual IEEE International Symposium on Personal, Indoor and Mobile Radio Communications, September 2014.

[7] A. Ahmadian, R. SivasivaGanesan, W. Zirwas, and B. Panzner, "Low complexity moore-penrose inverse for large CoMP areas with sparse massive MIMO channel matrices," in Proc. of the 27th Annual IEEE International Symposium on Personal, Indoor and Mobile Radio Communications, September 2016.

[8] R. SivasivaGanesan, W. Zirwas, B. Panzner, K. Pederson, and K. Valkealahti, "Integrating 3D channel model and grid of beams for 5G mMIMO system level simulations," in Proc. of the 84th IEEE Vehicular Technology Conference, September 2016.

[9] Study on Physical Layer Procedures, 3GPP Technical Report (TR)136.213, 2010. 